\title{
Distribution of human papillomavirus genotypes (2014-2016) in women with genital warts at a sexually transmitted disease clinic in Beijing, China
}

\author{
Liang Ma ${ }^{1}$, Shuang $\mathrm{Lu}^{1}$, Yongwei Jiang ${ }^{1}$, Min $\mathrm{Li}^{2}$, Xiao Cong ${ }^{1}$ \& Yongtong Cao*,1 \\ ${ }^{1}$ Department of Clinical Laboratory, China-Japan Friendship Hospital, Beijing 100029, PR China \\ ${ }^{2}$ Department of Obstetrics \& Gynecology, China-Japan Friendship Hospital, Beijing 100029, PR China \\ * Author for correspondence: Tel.: +86 1084205213; Fax: +86 10 84205213; caoyongtong1992@sina.com
}

\begin{abstract}
Aim: The aim of this study was to analyze the distribution of human papillomavirus (HPV) genotypes among women with genital warts in the Beijing region of China. Methods: A total of 350 women diagnosed with genital warts between 2014 and 2016 were analyzed by real-time polymerase chain reaction (PCR) to test for high-risk and low-risk HPV subtypes. Results: The results show that $52.6 \%(184 / 350)$ of cases were positive for HPV6+11. The three most common high-risk HPVs detected were HPV52, HPV16 and HPV58, detected in $12.0 \%$ (42/350), 10.6\% (37/350) and 10.0\% (35/350) of all cases, respectively. Conclusion: These results indicate that vaccines targeting HPV subtypes $6,11,16,52$ and 58 would have the greatest impact among sexually active women living in Beijing.
\end{abstract}

First draft submitted: 21 August 2017; Accepted for publication: 31 October 2017; Published online: 10 January 2018

Keywords: Beijing • China $\bullet$ distribution $\bullet$ genital warts $\bullet$ human papillomavirus $\bullet$ HPV $\bullet$ prevalence $\bullet$ sexually transmitted disease $\bullet$ subtype $\bullet$ vaccines $\bullet$ women

Human papillomaviruses (HPVs) are small double-stranded DNA viruses that belong to the family of papillomavirus [1]. There are as many as $170 \mathrm{HPV}$ genotypes have been identified [2], and approximately 40 kinds of these types specially infect the anogenital tract [3]. Genital warts are evident skin or mucosal growths in the anogenital area display as flat, papular or pedunculated growths on the genital mucosa [4]. Genital warts are one of the most common sexually transmitted diseases with a high prevalence $(0.5 \%)$ in Asia $[5,6]$. The disease results from infection with 'low-risk' HPV subtypes [7]. Infection with 'high-risk' HPV is associated with the development of precancerous lesions [8]. Treatments on genital warts are mainly divided into medications and surgery, medications include imiquimod, podophyllin, podofilox and trichloroacetic acid, surgery include freezing with liquid nitrogen, electrocautery, surgical excision and laser treatments $[9,10]$. Although the short-time effect of above treatment is good, the transmission rate of the related HPV infection is as high as $65 \%$, with a high risk of recurrence [7]. Thus, effective prevention against HPV transmission and infection is essential. Accordingly, many countries have implemented $\mathrm{HPV}$ vaccination programs.

A recent study by Chang et al. [11] indicates that the distribution of HPV subtypes differs among various regions throughout China. Comparison of other studies reporting on HPV prevalence in China indicates wide geographic variability in subtype distribution [12], thus undermining the selection of the most effective vaccine. Up to date, several vaccines have been developed to prevent HPV infection. Gardisil is a quadrivalent vaccine containing four genotypes, namely 16, 18, 6 and 11; and Cervarix (GSK) is a bivalent vaccine containing 16 and 18. In addition to providing excellent protection against the HPV types contained in the vaccine, Gardisil has also demonstrated significant cross-protection to other oncogenic types in both naive and previously infected women [13].

In order to identify the distribution of HPV subtypes in the Beijing area, samples collected from women with genital warts treated at a sexually transmitted disease clinic were examined. The results will contribute to the data 
Table 1. Human papillomavirus genotypes frequencies in genital warts.

\begin{tabular}{|c|c|c|c|c|c|c|c|c|}
\hline \multirow[t]{2}{*}{ Genotype } & \multirow[t]{2}{*}{ Single infection } & \multicolumn{5}{|c|}{ Multiple infection } & \multirow[t]{2}{*}{ Total } & \multirow[t]{2}{*}{$\%(95 \% \mathrm{Cl})$} \\
\hline & & 2 & 3 & 4 & 5 & 6 & & \\
\hline No. & 127 & 63 & 32 & 16 & 9 & 3 & 250 & \\
\hline$(\%)$ & $(36.6)$ & (17.7) & $(9.1)$ & $(4.5)$ & $(2.6)$ & $(0.8)$ & (71.4) & \\
\hline HPV $6+11^{\dagger}$ & 85 & 50 & 23 & 15 & 7 & 4 & 184 & $52.6(47.3-57.8)$ \\
\hline HPV 16 & 9 & 11 & 7 & 4 & 4 & 2 & 37 & $10.6(7.8-14.3)$ \\
\hline HPV 18 & 4 & 5 & 6 & 5 & 2 & 2 & 24 & $6.9(4.6-10.0)$ \\
\hline HPV 33 & 1 & 2 & 2 & 2 & 2 & 1 & 10 & $2.9(1.5-5.2)$ \\
\hline HPV 35 & 0 & 2 & 4 & 1 & 2 & 0 & 9 & $2.6(1.3-4.9)$ \\
\hline HPV 39 & 2 & 5 & 12 & 5 & 5 & 0 & 29 & $8.3(5.8-11.7)$ \\
\hline HPV 45 & 2 & 3 & 0 & 1 & 1 & 0 & 7 & $2.0(1.0-4.0)$ \\
\hline HPV 51 & 1 & 9 & 7 & 5 & 5 & 3 & 30 & $8.6(6.1-12)$ \\
\hline HPV 68 & 3 & 2 & 10 & 0 & 1 & 0 & 16 & $4.6(2.8-7.3)$ \\
\hline
\end{tabular}

on HPV genotype-specific prevalence in patients with genital warts in order to encourage the implementation of an HPV vaccine program in Beijing, China.

\section{Materials \& methods}

\section{Patients \& sample collection}

This was a retrospective study of 350 women diagnosed with genital warts who were treated at the Sexually Transmitted Disease Clinic in Beijing, China between September 2014 and August 2016. Genital epithelial cells and condyloma tissues were scraped and transferred using a liquid-based media for further analysis. The patients' confidentiality was protected by hiding their identities. Informed consent was obtained from all the patients prior to their enrollment in the study. The study design adhered to the principles of the Helsinki Declaration and was approved by the ethics committees of our hospital.

\section{HPV subtype genotyping}

DNA from the genital epithelial cells and warts tissues was prepared according to a previous publication [14]. A Chinese State Food and Drug Administration-approved HPV genotyping assay (Liferiver, Shanghai, China) was used to detect HPV genotypes by real-time PCR. This test can individually detect 13 high-risk HPV subtypes (16, $18,31,33,35,39,45,51,52,56,58,59$ and 68), and the combined low-risk subtypes $(6+11)$ [14].

\section{Statistical analysis}

The distribution of HPV subtypes was expressed as the percentage of HPV-positive specimens among all cases of genital warts with 95\% CI using MetaAnalyst Beta 3.0 software. The association between HPV subtype and patient age was assessed by the $\chi^{2}$ test performed with SAS9.3 software. A p value of $\leq 0.05$ was considered statistically significant.

\section{Results}

Characterization of HPV genotypes in women with genital warts

Among 350 cases of genital warts, 13 types of high-risk HPV DNA and $6+11$ types were detected in 250 of them, for an overall prevalence of $71.4 \%$ (Table 1). In the HPV-positive patients, the single HPV genotype infection was 36.6\%, and the multiple HPV genotypes infection was 35.0\%. Overall, HPV6+11 were found in 52.6\% (184/350) of cases. Of the 13 most common high-risk HPV genotypes, HPV52 was detected in 12.0\% (42/350), HPV16 in $10.6 \%(37 / 350)$ and HPV58 in 10.0\% (35/350) of genital warts cases. 


\begin{tabular}{|c|c|c|c|c|}
\hline Genotype & \multicolumn{2}{|c|}{ HPV $6+11$ positive $(n=184)$} & \multicolumn{2}{|c|}{ HPV6+11 negative $(n=166)$} \\
\hline HPV 16 & 18 & $9.8(6.3-15.0)$ & 19 & $11.4(7.4-17.2)$ \\
\hline HPV18 & 15 & $8.1(5.0-13.1)$ & 9 & $5.4(2.8-10.1)$ \\
\hline HPV31 & 9 & $4.9(2.6-9.1)$ & 5 & $3.0(1.3-7.0)$ \\
\hline HPV35 & 7 & $3.8(1.8-7.8)$ & 2 & $1.2(0.3-4.7)$ \\
\hline HPV 39 & 15 & $8.2(5.0-13.1)$ & 14 & $8.4(5.1-13.7)$ \\
\hline HPV45 & 3 & $1.6(0.5-4.9)$ & 2 & $1.2(0.3-4.7)$ \\
\hline HPV51 & 27 & $14.6(10.3-20.6)$ & 3 & $1.8(0.6-5.5)$ \\
\hline HPV52 & 28 & $15.2(10.7-21.2)$ & 14 & $8.4(5.1-13.7)$ \\
\hline HPV68 & 5 & $2.7(1.1-6.4)$ & 11 & $6.6(3.7-11.6)$ \\
\hline
\end{tabular}

Table 3. Low-risk and high-risk infection according to age and correlation between infection and age.

\begin{tabular}{|c|c|c|c|c|c|c|c|}
\hline Genotype & $<20$ & $21-25$ & $26-30$ & $31-35$ & $36-40$ & $>41$ & Total \\
\hline $6+11$ & 4 & 22 & 23 & 18 & 6 & 12 & 85 \\
\hline $\mathrm{HR}^{\ddagger}$ & 2 & 25 & 25 & 6 & 4 & 4 & 66 \\
\hline Total & $14(4.0 \%)$ & $109(31.1 \%)$ & $114(32.6 \%)$ & $59(16.9 \%)$ & $22(6.3 \%)$ & $32(9.1 \%)$ & $350(100 \%)$ \\
\hline
\end{tabular}

$\chi^{2}$ test for different between ages $\left(\chi^{2}=18.20, p=0.25\right.$.

${ }^{\dagger} 6+11 /$ HR: $6+11$ co-infected with HR HPV.

$\ddagger$ HR: HR HPV infected only.

§Others: undetected HPV DNA.

HR: High risk; HPV: Human papillomavirus.

Among the 250 detected HPV-positive cases, 127 (50.8\%) were infections of a single HPV subtype, including HPV6+11 $(\mathrm{n}=85)$, HPV16 $(\mathrm{n}=9)$ and HPV52 $(\mathrm{n}=8)$ (Table 1). For the remaining HPV-positive cases, 62/250 (24.8\%) were infected with two HPV subtypes, 32/250 (12.8\%) contained three HPV subtypes, 16/250 (6.4\%) had four subtypes, 9/250 (3.6\%) had five subtypes and six HPV subtypes were detected in 3/250 (1.2\%) cases.

Table 2 shows the prevalence of associated high-risk infections in HPV6+11 positive and negative cases. The three most common high-risk subtypes co-infected with HPV6+11 were HPV52 (15.2\%; 95\% CI: 10.7-21.2\%), HPV51 (14.6\%; 95\% CI: 10.3-20.6\%) and HPV58 (13.6\%; 95\% CI: 9.3-19.3\%). In contrast, HPV16 (11.4\%; 95\% CI: 7.4-17.2\%), HPV39 (8.4\%; 95\% CI: 5.1-13.7\%) and HPV52 (8.4\%; 95\% CI: 5.1-13.7\%) were most common in HPV6+11-negative genital warts cases.

\section{Prevalence of HPV according to age groups}

The ages of the 350 patients with genital warts ranged from 16 to 79 years old. Among these, the vast majority $(80.9 \%)$ of patients were between 21 and 35 years of age. The prevalence of infection with the various HPV subtypes according to patient age is presented in Table 3. No significant differences were observed.

\section{Discussion}

This study provides an assessment of the prevalence of HPV6+11 and 13 high-risk HPV genotypes isolated from female patients with genital warts in Beijing. The overall prevalence of HPV infection in genital warts was $71.4 \%$, which is lower than the reported overall HPV prevalence (84.2-88.7\%) in China [12]. It is not surprising that not all the HPV subtypes were detected in this study. The results show that the most common infection is from the 
low-risk HPV6+11, which was detected in approximately half of the genital warts cases tested. According to the study by Chang et al. [11], HPV6+11 prevalence among individuals with genital warts is $65.2 \%$ in central areas of China. Other reports show wide variability in infection rates of HPV6 or 11, ranging from 53.6 to $96.4 \%$ [12,15,16]. Thus, confirmatory studies are needed to more firmly establish the prevalence of HPV6+11 in different geographic regions in China.

The second most common HPV subtype detected was HPV52, which was found in $12.0 \%$ of cases. HPV16 was detected in $10.6 \%$ cases of genital warts cases in the present study, which is in agreement with the overall prevalence of HPV16 in China (10.4\%) reported by Chang et al. [11]. The prevalence of HPV58 was similar, detected in $10.0 \%$. This is the first report detailing the prevalence of HPV52 and HPV58 in genital warts. Furthermore, the fact that the prevalence of HPV16 and HPV58 were common in this study is valuable important, as these subtypes are associated with cervical cancer in Chinese women [17-19].

The results of this study indicate that $35.0 \%$ of women with genital herpes have multiple HPV-subtype infections, a prevalence that is higher than that reported by Chang et al. [11]. However, this rate falls within the range reported from studies in other countries, where the multiple infection rates in genital warts is $28.3-68.3 \%[17,20,21]$. The results of the present study show that the proportion of cases with HPV6+11 co-infected with HPV 52, 51 or 58 was higher than those co-infected with HPV16, suggesting that the vaccine development for Beijing patients should focus on the combination of these subtypes among women with genital warts. Based on our data, the high-risk HPVs are also highly prevalent in the population with genital warts, this result may be associated with the spread of HPV infection. HPV vaccination program in China may reduce the burden of disease associated with genital warts and achieve performance as good as in western countries. However, based on the results of our study and others, there is a variation of HPV prevalence by geographic regions. Therefore, the effectiveness of a vaccine in reduction of disease burden may differ by population, and the vaccine concluding more HPV subtypes is needed in Beijing, such as 52, 51, 58, 33 and so on.

A cross-sectional study of 18,498 sexually active women 15-74 years of age from the general population of 15 areas in four continents showed that HPV prevalence peaks at approximately $25-35$ years of age [22]. Indeed, patients in the present study were generally within this age range, with roughly a third between 21 and 25 years of age and another third between 26 and 30 years. Moreover, a prophylactic quadrivalent HPV6/11/16/18 vaccine has shown high efficacy, safety and tolerability in young Asia-Pacific women [23], though whether this is a cost-effective way for reducing HPV-associated genital warts needs further study. A nonavalent vaccine, which additionally targets HPV31/33/45/52/58, is currently undergoing Phase III clinical trials with the goal of establishing $15-30 \%$ higher anticancer effects [24]. This vaccine may be a good candidate for trials in Beijing, as it incorporates the most common HPV subtypes identified in this study.

The present study was performed with 350 patients with women diagnosed with genital warts between 2014 and 2016, which would identify the most common HPV subtypes among women with genital warts in this region. However, there are still some limitations. First, this study was limited to patients from only one hospital in the Beijing area, and more samples need to be detected in this area. Second, a Chinese State Food and Drug Administration-approved HPV genotyping assay (Liferiver, Shanghai, China) was used to detect HPV genotypes by real-time PCR. This test only can individually detect 13 high-risk HPV subtypes $(16,18,31,33,35,39,45$, $51,52,56,58,59$ and 68$)$, and the combined low-risk subtypes $(6+11)$, and the other HPV subtypes cannot be detected.

\section{Conclusion}

In conclusion, the study has reported on the first study of HPV genotype distributions in genital warts patients in Beijing, China. The data suggested women with genital warts in Beijing have more multiple HPV-subtype infections. These results provide a basis for future studies evaluating the vaccines that can be most effective for this population.

\section{Future perspective}

About 30-40 HPV types regularly or sporadically infect the mucosal surfaces of the anogenital tract. Although current opinion believe that the 90\% genital warts are caused by two low-risk HPV subtypes 6 and 11 [25], the real situation regarding the prevalence and genotype distribution of low-risk HPV in populations with genital warts is rarely reported. There is variation of HPV prevalence by geographic regions, sexes and age groups in China, and the distribution of women HPV subtypes in urban areas is different in rural areas [11]. HPV vaccines are sub-unit 
vaccines consisting of virus-like particles) made of one protein - the major HPV coat or capsid protein L1.Gardasil 9 is a nine-valent (nHPV) 6, 11, 16, 18, 31, 33, 45, 52, 58 virus-like particle vaccines from Merck Sharp \& Dohme licensed by the US FDA in December 2014 for use in 9-26 year old females and 9-15 year old boys. To achieve optimal vaccine effectiveness immunization ideally should be completed before the start of sexual activity. HPV vaccination programs have now been introduced in many countries. The study about the distribution of HPV subtypes could play an important role in genital warts prevention.

\section{Executive summary}

- Genital warts are one of the most common sexually transmitted diseases among young women worldwide that are caused in part by low-risk human papillomavirus (HPV) infection.

- However, the distribution of HPV genotypes varies by geographic location, and more data are needed regarding the prevalence of genotypes in the Beijing region of China.

Methods

- This was a retrospective study of 350 women diagnosed with genital warts who were treated at the Sexually Transmitted Disease Clinic in Beijing, China between September 2014 and August 2016.

- DNA from the genital epithelial cells and warts tissues was prepared, and HPV genotyping assay (Liferiver, Shanghai, China) was used to detect HPV genotypes by real-time PCR. This test can individually detect 13 high-risk HPV subtypes $(16,18,31,33,35,39,45,51,52,56,58,59$ and 68), and the combined low-risk subtypes $(6+11)$.

- The distribution of HPV subtypes was expressed as the percentage of HPV-positive specimens among all cases of genital warts with $95 \% \mathrm{Cl}$ using MetaAnalyst Beta 3.0 software. The association between HPV subtype and patient age was assessed by the $\chi^{2}$ test performed with SAS9.3 software. A p-value of $\leq 0.05$ was considered statistically significant.

Results

- Among 350 cases of genital warts, 13 types of high-risk HPV DNA and $6+11$ types were detected in 250 of them, for an overall prevalence of $71.4 \%$.

- The results show that $52.6 \%(184 / 350)$ of cases were positive for HPV6+11. The three most common high-risk HPVs detected were HPV52, HPV16 and HPV58, detected in 12.0\% (42/350), 10.6\% (37/350) and 10.0\% (35/350) of all cases, respectively.

- Of the 250 HPV-positive cases, 49.2\% (123/250) were infected by multiple HPV subtypes. Cases infected with HPV6+11 were most commonly co-infected with HPV51, HPV52 and HPV58, whereas HPV16, HPV39 and HPV52 were more common among HPV6+11-negative cases.

Conclusion

- These results indicate that vaccines targeting HPV subtypes $6,11,16,52$ and 58 would have the greatest impact among sexually active women living in Beijing.

\section{Acknowledgements}

The authors thank ZY Wen, at the Center for Clinical Epidemiology and Biostatistics, China-Japan Friendship Hospital, China.

\section{Open access}

This work is licensed under the Attribution-NonCommercial-NoDerivatives 4.0 Unported License. To view a copy of this license, visit http://creativecommons.org/licenses/by-nc-nd/4.0/

Financial \& competing interests disclosure

This study was supported by the National Major Scientific Instruments and Equipment Development Projects of China (grant no: 2012YQ030261). The authors have no other relevant affiliations or financial involvement with any organization or entity with a financial interest in or financial conflict with the subject matter or materials discussed in the manuscript apart from those disclosed. No writing assistance was utilized in the production of this manuscript. 


\section{References}

Papers of special note have been highlighted as: $\bullet$ of interest; $\bullet \bullet$ of considerable interest

1 Woodman CB, Collins SI, Young LS. The natural history of cervical HPV infection: unresolved issues. Nat. Rev. Cancer 7(1), 11-22 (2007).

- States the natural history of cervical human papillomavirus (HPV) infection.

2 Ghiyyoni R, Accardi R, Chiocca S, Tommasino M. Role of human papillomaviruses in carcinogenesis. Ecancermedicalscience 9, 526 (2015).

- States the role of HPV in carcinogenesis.

3 de Villiers EM, Fauquet C, Broker TR, Bernard HU, zur Hausen H. Classification of papillomaviruses. Virology 324(01), 17-27 (2004).

- States the classification of papillomaviruses.

4 Steben M, Garland SM. Genital warts. Best Pract. Res. Clin. Obstet. Gynaecol. 28(7), 1063-1073 (2014).

5 Parkin DM, Louie KS, Clifford G. Burden and trends of type-specific human papillomavirus infections and related diseases in the Asia Pacific region. Vaccine 26(12), M1-M16 (2008).

6 Lynde C, Vender R, Bourcier MB, Bhatia N. Clinical features of external genital warts. J. Cutan. Med. Surg. 17(Suppl. 2), S55-S60 (2013).

7 Bhatia N, Lynde C, Vender R, Bourcier M. Understanding genital warts: epidemiology, pathogenesis, and burden of disease of human papillomavirus. J. Cutan. Med. Surg. 17(Suppl. 2), S47-S54 (2013).

8 Paavonen J. Human papillomavirus infection and the development of cervical cancer and related genital neoplasias. Int. J. Infect. Dis. 11(Suppl. 2), S3-S9 (2007).

9 Leszczyszyn J, Łebski I, Łysenko L, Hirnle L, Gerber H. Anal warts (condylomata acuminata) - current issues and treatment modalities. Adv. Clin. Exp. Med. 23(2), 307-311 (2014).

10 Fathi R, Tsoukas MM. Genital warts and other HPV infections: established and novel therapies. Clin. Dermatol. 32(2), 299-306 (2014).

- Demontrates genital warts and other HPV infections.

11 Chang L, Ci P, Shi J et al. Distribution of genital wart human papillomavirus genotypes in China: a multi-center study. J. Med. Virol. 85(10), 1765-1774 (2013).

-. First demontrates the distribution of genital wart HPV genotypes in China.

12 Wand H, Qiao YL. Human papillomavirus type-distribution in condylomata acuminata of mainland China: a meta-analysis. Int. J. STD AIDS 19(10), 680-684 (2008).

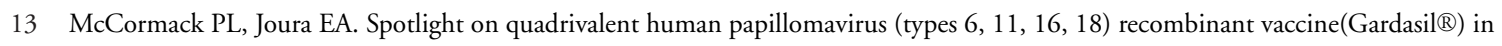
the prevention of premalignant genital lesions, genital cancer, and genital warts in women. BioDrugs 25(5), 339-343 (2011).

14 Lu S, Cong X, Li M, Chang F, Ma L, Cao YT. Distribution of high-risk human papillomavirus genotypes in HPV-infected women in Beijing, China. J. Med. Virol. 87(3), 504-507 (2015).

- Demonstrates the distribution of high-risk human papillomavirus genotypes in HPV-infected women in Beijing, China.

15 Potocnik M, Kocjan B, Seme K, Poljak M. Distribution of human papillomavirus (HPV) genotypes in genital warts from males in Slovenia. Acta Dermatovenerol. Alp. Panonica. Adriat. 16(03), 91-96, 98 (2007).

16 Garland SM, Steben M, Sings HL et al. Natural history of genital warts: analysis of the placebo arm of 2 randomized Phase III trials of a quadrivalent human papillomavirus (types 6, 11, 16, and 18) vaccine. J. Infect. Dis. 199(06), 805-814 (2009).

-• Demonstrates the effect of quadrivalent human papillomavirus (types 6, 11, 16 and 18) vaccine.

17 Chan PK, Lam CW, Cheung TH et al. Association of human papillomavirus type 58 variant with the risk of cervical cancer. J. Natl Cancer Inst. 94(16), 1249-1253 (2002).

18 Han-liang J, Su Z, Lin-fu Z. [Human papillomavirus infection in women with cervical lesion of Huzhou area of Zhejiang province]. Zhejiang Da Xue Xue Bao Yi Xue Ban 36(06), 549-552 (2007).

19 Liu SS, Tsang PC, Chan KY et al. Distribution of six oncogenic types of human papillomavirus and type 16 integration analysis in Chinese women with cervical precancerous lesions and carcinomas. Tumour Biol. 29(02), 105-113 (2008).

20 Nielson CM, Harrris RB, Flores R et al. Multiple-type human papillomavirus infection in male anogenital sites: prevalence and associated factors. Cancer Epidemiol. Biomarkers Prev. 18(04), 1077-1083 (2009).

21 Nielson A, Munk C, Jørgensen HO, Winther JF, van den Brule AJ, Kjaer SK. Multiple-type human papillomavirus infection in younger uncircumcised men. Int. J. STD AIDS 24(2), 128-133 (2013).

22 Franceschi S, Herrero R, Clifford GM et al. Variations in the age-specific curves of human papillomavirus prevalence in women worldwide. Int. J. Cancer 119(11), 2677-2684 (2006).

-• Demonstrates the variations in the age-specific curves of HPV prevalence in women worldwide.

23 Tay EH, Garland S, Tang G et al. Clinical trial experience with prophylactic HPV 6/11/16/18 VLP vaccine in young women from the Asia-Pacific region. Int. J. Gynaecol. Obstet. 102(03), 275-283 (2008). 
24 Perez J. For cancers caused by HPV, two vaccines were just the beginning. J. Natl Cancer Inst. 103(5), 360-362 (2011).

25 Derkay CS, Wiatrak B. Recurrent respiratory papillomatosis: a review. Laryngoscope 118(07), 1236-1247 (2008). 
